\title{
Avaliação da contribuição dos dados hemogasométricos pré extubação e na retirada da ventilação mecânica
}

\author{
Evaluation of the contribution of pre-extubation hemogasometric data and mechanical \\ ventilation withdrawal
}
Evaluación de la contribución de los datos hemogasométricos pre extuba y en la retirada de la ventilación mecánica

Jonas Davi Heiderick Mota ${ }^{1 *}$, Yuri de Souza Rodrigues', Flávia dos Santos Lugão de Souza².

\section{RESUMO}

Objetivo: Analisar a importância dos dados gasométricos no processo de extubação e na retirada da ventilação mecânica de pacientes em uma unidade de tratamento intensivo de um hospital da Zona da Mata Mineira. Método: Pesquisa documental, retrospectiva dos meses de agosto a dezembro de 2018, de carácter descritivo. Resultados: $\mathrm{Na}$ amostragem inicial foram analisadas 75 amostras de controle de desmame, sendo excluídos 20 de pacientes que não passaram no teste de respiração espontânea. Assim a amostra foi realizada com 55 pacientes em tratamento intensivo, sendo 31 homens $(56,4 \%)$ e 24 mulheres $(43,6 \%)$ com idade mínima de 13 anos e máxima 88 anos. Conclusão: Foi constatado que os valores de PAO2 e PCO2 da gasometria arterial no processo de desmame não interferem no sucesso da retirada da VMI. Os dados hemogasométricos contribuem de forma positiva no processo de desmame e retirada da ventilação mecânica, fazendo parte da avaliação para a decisão da retirada ou cancelamento do Teste de respiração espontânea (TRE) e retorno para a ventilação artificial, além de predizer a necessidade de Ventilação Não Invasiva (VNI) pós extubação nos pacientes retentores de PCO2 no momento do TRE.

Palavras-chave: Gasometria, Desmame da Ventilação Mecânica, Extubação.

\begin{abstract}
Objective: To analyze the importance of gasometric data in the extubation process and in the withdrawal of mechanical ventilation from patients in an intensive care unit of a hospital in the Zona da Mata Mineira. Method: Documentary research, retrospective from August to December 2018, of a descriptive nature. Results: In the initial sampling, 75 weaning control samples were analyzed, and 20 were excluded from patients who did not pass the spontaneous breathing test. Thus, the sample consisted of 55 intensive care patients, 31 men $(56.4 \%)$ and 24 women (43.6\%), with a minimum age of 13 years and a maximum of 88 years. Conclusion: It was observed that the PAO2 and PCO2 values of arterial blood gas in the weaning process did not interfere in the successful withdrawal of IMV. Hemogasometric data contribute positively to the weaning process and withdrawal of mechanical ventilation, being part of the evaluation for the decision to withdraw or cancel the Spontaneous Breathing Test (RET) and return to artificial ventilation, in addition to predicting the need for Ventilation Non-invasive (NIV) after extubation in PCO2-retained patients at the time of ERT.
\end{abstract}

Key words: Gasometry, Mechanical Ventilation Weaning, Extubation.

${ }^{1}$ Faculdade do Futuro (FAF), Manhuaçu-MG. *E-mail: jdheiderick1@gmail.com

2Universidade Federal do Rio de Janeiro (UNIRIO), Rio de Janeiro-RJ.

SUBMETIDO EM: 4/2019

ACEITO EM: 5/2018

PUBLICADO EM: 7/2019

REAS/EJCH | Vol. 11(12) | e749 | DOI: https://doi.org/10.25248/reas.e749.2019 Página 1 de $\mathbf{9}$ 


\section{RESUMEN}

Objetivo: Analizar la importancia de los datos gasométricos en el proceso de extubación y en la retirada de la ventilación mecánica de pacientes en una unidad de tratamiento intensivo de un hospital de la Zona de la Mata Minera. Método: Investigación documental, retrospectiva de los meses de agosto a diciembre de 2018, de carácter descriptivo. Resultados: En el muestreo inicial se analizaron 75 muestras de control de destete, siendo excluidos 20 de pacientes que no pasaron la prueba de respiración espontánea. La muestra fue realizada con 55 pacientes en tratamiento intensivo, siendo 31 hombres $(56,4 \%)$ y 24 mujeres $(43,6 \%)$ con edad mínima de 13 años y máxima 88 años. Conclusión: Se constató que los valores de PAO2 y PCO2 de la gasometría arterial en el proceso de destete no interfieren en el éxito de la retirada de la VMI. Los datos hemogasométricos contribuyen de forma positiva en el proceso de destete y retirada de la ventilación mecánica, haciendo parte de la evaluación para la decisión de la retirada o cancelación del Test de respiración espontánea (TRE) y retorno para la ventilación artificial, además de predecir la necesidad de Ventilación No Invasiva (VNI) después de la extubación en los pacientes retenedores de PCO2 en el momento del TRE.

Palabras clave: Gasometría, Desmame de la Ventilación Mecánica, Extubación.

\section{INTRODUÇÃO}

A utilização da respiração artificial é um procedimento rotineiro nos centros e unidades de terapia intensiva (UTI's), estudos demonstram que $31 \%$ a $46 \%$ dos pacientes admitidos nessas unidades utilizam Ventilação Mecânica (VM) em algum momento durante a internação (PIOTTO RF, et al., 2011).

A maioria dos pacientes submetidos ao suporte ventilatório mecânico pode ser rapidamente retirada do ventilador, assim que as condições respiratórias e de base tenha sido tratada e/ou estabilizada. $O$ prolongamento desnecessário desse processo pode acarretar o aumento dos custos hospitalares e as complicações associadas à VM (SAUGEL B, et al., 2018).

Para Laizo A, et al. (2010) cerca de $5 \%$ a $30 \%$, dos pacientes tem dificuldade no desmame, não sendo possível a remoção do ventilador nas primeiras tentativas. Este fato acontece mais em portadores de doenças pulmonares prévias, cardiopatias, grandes cirurgias abdominais ou torácicas, tempo prolongado de ventilação mecânica, disfunção de múltiplos órgãos e doenças neurológicas com prognóstico prolongado (PIOTTO RF, et al., 2011; CASSEL LW e VIEIRA SRR, 2013).

O desmame é definido como uma mudança da ventilação mecânica para a espontânea, havendo, porém, variação na forma de administrar esse processo, o qual pode ser classificado como rápido ou prolongado. Este processo é seguido de avaliações clinicas e de exames complementares para que seja realizado com sucesso Pinto JMA, et al. (2017); Rolim JFC, et al. (2011) contribuem relatando que a retirada da prótese ventilatória deve ser realizada o mais precocemente possível, pois longos períodos em ventilação mecânica invasiva podem gerar complicações graves, como infecções, barotraumas, fraqueza e incoordenação dos músculos respiratórios.

Portanto, é necessário a utilização de recursos para melhor predizer o sucesso do desmame e reduzir os riscos ao paciente, como por exemplo, a avaliação dos gases sanguíneos e valores hemogasométricos (PAREDES ER, et al., 2013).

Alguns estudos buscam determinar o melhor momento para retirada do tubo. Quando o suporte ventilatório pode ser retirado, a decisão de extubar deve ser tomada (LAIZO A, et al., 2010). Entre 5\% a $25 \%$ dos pacientes extubados têm a probabilidade de retornar para prótese ventilatória e ser restituída a ventilação mecânica independente da técnica.

A falha na extubação pode prolongar a permanência hospitalar, aumentar a frequência de traqueostomia e complicações pulmonares, piorar os resultados funcionais e aumentar a mortalidade, que em pacientes reintubados é alta chegando a $43 \%$ e $12 \%$ naqueles com sucesso na extubação (JEGANATHAM N, et al., 2015).

REAS/EJCH | Vol. 11(12) | e749 | DOI: https://doi.org/10.25248/reas.e749.2019 Página 2 de 9 
Reis HFC, et al. (2013) cita ainda algumas intercorrências associadas ao processo de reintubação, como parada cardiorrespiratória, arritmias e pneumonia aspirativa, que podem ocorrer em até $28 \%$ dos pacientes (FREITAS EEC e DAVID CMN, 2006).

Dentre estes pacientes que necessitam de reintubação também há um risco aumentado de desenvolver pneumonia e sepse. Devemos intensificar avaliação a beira do leito e exames complementares e valores hemogasométricos para que a extubação seja executada da forma mais segura possível (SILVA GJP, et al., 2010).

Os dados hemogasométricos são colhidos através de um exame de gasometria arterial, um exame invasivo que avalia as pressões parciais de oxigênio (pO2), gás carbônico (pCO2), e determinação do valor de $\mathrm{pH}$ sanguíneo. Com essas dosagens são obtidos outros parâmetros que são calculados, como bicarbonato (HCO3-), saturação de oxigênio, excesso de base, entre outros. Estes dados nos fornecem informações essenciais para avaliação e tratamento de doenças que afetam as trocas gasosas e o equilíbrio ácido-base.

O exame consiste em uma punção de sangue arterial que pode ser obtida pela artéria radial, braquial ou femoral. O local de punção deve ser esterilizado previamente e puncionado com uma seringa e agulha própria, a fim de obter 2 a $3 \mathrm{~mL}$ de sangue, a seringa mais indicada hoje em dia é a de plástico polipropileno de alta densidade, que são descartáveis, eliminando assim contaminação cruzada. Além disso, possuem custo menor, sendo mais convenientes e não correm risco de quebrar (PINTO JMA, et al., 2017; VIESSER L, 2012).

Avaliar os gases sanguíneos e as trocas gasosas está diretamente associado ao desmame do paciente em ventilação mecânica invasiva e a sua extubação. Estando assim indicada para ser realizada antes do procedimento de extubação como um dos parâmetros que pode predizer o sucesso do mesmo. De acordo com o Manual Prático de Medicina Intensiva os valores de referência para a gasometria arterial são: $\mathrm{pH}: 7,35$ a 7,45; $\mathrm{pCO}_{2}$ : 35 a $45 \mathrm{mmHg} ; \mathrm{pO}_{2}: 80$ a $100 \mathrm{mmHg}$; BE: +/- 2 mmol/L; $\mathrm{HCO}_{3}: 22$ a $28 \mathrm{mmol} / \mathrm{L}$; Saturação de O2: >95\% (CALDEIRA FM e WESTPHAL GA, 2006).

Tendo em vista o exposto, a seguinte pesquisa documental teve como objetivo avaliar a contribuição dos dados hemogasométricos pré extubação, em uma unidade de tratamento intensivo de um hospital da zona da mata mineira, com o intuito de melhorar a atenção ao paciente no momento da retirada da ventilação artificial, fazendo com que o procedimento seja realizado com maior segurança, menor risco de reintubação e danos para o paciente.

\section{MÉTODOS}

Trata-se de uma pesquisa documental, retrospectiva, de carácter descritivo onde a coleta de dados foi realizada nos documentos e indicadores da Fisioterapia em um Centro de Terapia Intensiva (CTI) do Hospital Cezar Leite, na cidade de Manhuaçu (MG).

Foram utilizados artigos dos anos de 2006 a 2018, buscados através das bases de dados da Biblioteca Virtual em Saúde (BVS) selecionando artigos do Scielo, Lilacs, Bdenf e Medline. Foram excluídos os artigos que não abordavam o assunto e aqueles fora do corte temporal, com isso obtivemos 20 artigos.

\section{Sujeitos da amostra}

A pesquisa foi realizada com os pacientes que preencheram critérios de desmame da ventilação mecânica invasiva, teste de respiração positivo seguido de extubação orotraqueal ou retirada da VM, sendo excluídos os que não tinham realizado exame de gasometria arterial e que não passaram no teste de respiração espontânea mais de três vezes.

\section{Instrumentos de coleta de dados}

O instrumento para a coleta de dados utilizado no estudo é um documento padrão preenchido pela equipe de profissionais da fisioterapia que faz parte do procedimento Sistêmico PRS. EMA9 para admissão e controle do desmame da ventilação mecânica invasiva. Todos os pacientes que dão entrada no CTI em VMI ou que são intubados no setor e que progridem para o desmame da prótese ventilatório TOT ou TQT são preenchidos 
estes controles. A liberação destes dados para pesquisa é realizada através da autorização da direção e coordenação de fisioterapia. Os dados foram coletados no ano de 2018 entre o período de agosto a dezembro. Os critérios de inclusão: pacientes que foram Intubados no Setor CTI, provenientes de outras instituições, Unidades de Pronto Atendimento (UPA's) da cidade local, internos de outros setores hospitalares e do Pronto Atendimento (PA), destes foram selecionados todos que estavam em ventilação mecânica e que passaram no o teste de respiração espontânea, progredindo para extubação orotraqueal ou retirada da ventilação mecânica (VM).

Não foi realizado exclusão por diferença de diagnóstico ou idade pois não modifica o objetivo da pesquisa, tendo em vista que todos pacientes foram extubados após passar por testes e exames complementares. As gasometrias são coletadas pela equipe de enfermagem e inseridas no aparelho hemogasômetro GEM PREMIER 3.500 dentro de um tempo máximo de $5 \mathrm{~min}$, as amostras que ultrapassam esse tempo são descartadas e é colhida nova amostragem de sangue arterial.

Para a análise da gasometria, a amostra utilizada é sangue total, sendo necessário que a amostra demonstre ausência completa de coágulos, pois, além de obstruir a passagem de amostra em analisadores de gases sanguíneos, uma amostra com micro coágulos não é homogênea, com isso o resultado de sua análise não será exato, podendo ser analisado o plasma ou células sanguíneas causando resultados falsos. Assim, a amostra deve ser avaliada pelos profissionais responsáveis. O teste de respiração espontânea e a extubação seguem os seguintes critérios:

\section{1 - Teste de Respiração Espontânea (TRE)}

São avaliados os pacientes 3 vezes ao dia (manhã e tarde e noite) pela equipe de fisioterapia, os que preencherem os critérios de reversão da insuficiência respiratória devem ser submetidos a um TRE, que deve ser feito colocando uma PSV de 5 a $7 \mathrm{cmH} 20$ ou em Peça T por um período de 30 min a 120min, se o teste for positivo, colhe-se nova gasometria arterial, parâmetros normais, Glasgow > 10 seguir com extubação orotraqueal.

\section{2 - Extubação Orotraqueal}

Verificar se a dieta nasoenteral foi suspensa posicionando o paciente no leito em decúbito dorsal com cabeceira elevada à 45 graus, após, aspirar o TOT e as vias aéreas superiores (VAS) do paciente, desinsuflar o cuff com seringa, solicitar ao paciente abrir cavidade oral e realizar uma inspiração profunda seguida de tosse, retirar o TOT sem ser abruptamente para não lesionar vias aéreas, instalar o dispositivo de oxigenoterapia eleita (máscara de Venturi ou cateter binasal de O2), ofertando uma concentração de O2 suficiente $\mathrm{SpO} 2 \geq 93 \%$, solicitar uma tosse espontânea, realizar a ausculta pulmonar e das VAS para descartar laringo espasmos e broncoespasmos, monitorizar os sinais vitais, saturação parcial de oxigênio (SpO2), padrão ventilatório, expansibilidade torácica e o nível de consciência.

\section{Ética na Pesquisa}

A presente pesquisa não necessitou de aprovação do Comitê de Ética em Pesquisas (CEP), uma vez que, conforme a Resolução oㅜ 510, de 07 de abril de 2016, em seu parágrafo único, inciso V, prevê que "pesquisa com bancos de dados, cujas informações são agregadas, sem possibilidade de identificação individual" não serão registradas nem avaliadas pelo sistema CEP/CONEP.

\section{Tratamento estatístico dos dados}

Os dados coletados foram digitados no programa Excel for Windows 10 (Microsoft Office Professional Plus 2016) e, posteriormente avaliado pelos autores as médias e desvio padrão. Também foi realizado o teste $T$ de Student em algumas amostras e o nível de significância estatística adotado para este teste foi de $5 \%$, ou seja, o valor de $P$ igual ou inferior a 0,05 para resultados estatisticamente significativos $(p<0,05)$.

\section{RESULTADOS}

Para melhor elucidar os resultados desta pesquisa separamos os dados em dois grupos diferentes: 
Grupo 1 - pacientes intubados que progrediram para extubação orotraqueal $(n=35),(63,6 \%$ com tubo orotraqueal).

Grupo 2 - pacientes traqueostomizados que progrediram para retirada da VM ( $n=20),(36,4 \%$ com traqueostomia).

$\mathrm{Na}$ amostragem inicial foram analisadas 75 amostras de controle de desmame, sendo excluídos 20 de pacientes que não passaram no teste de respiração espontânea. Assim a amostra foi realizada com 55 pacientes em tratamento intensivo, sendo 31 homens (56,4\%) e 24 mulheres (43,6\%) com idade mínima de 13 anos e máxima 88 anos.

A realização dos desmames foi de caráter rápido, ou seja, em menos de $24 \mathrm{~h}$ em $100 \%$ ( $n=35)$ dos pacientes do grupo 1. No grupo 2, os desmames de caráter prolongado somaram $100 \%(n=20)$.

A utilização da peça T como teste de respiração espontânea prevaleceu em 91,4\% ( $n=32)$ no grupo 1, e $80 \%(n=16)$ no grupo 2.

Da amostragem total $(n=55)$ a taxa de sucesso da retirada da VM foi de $76,36 \%(n=42)$ (Tabela 1). A taxa de reintubação foi de 14,2\% ( $n=5)$ nos pacientes do grupo 1, corroborando com a literatura vigente (LIU $L$, et al., 2012; MABROUK AA, et al., 2015; CASSEL LW e VIEIRA SRR, 2013; ROLIM JFC, et al., 2011).

Ainda no grupo 1, em 3 dos 5 casos com falha no desmame a reintubação foi necessária devido a disfunções do sistema respiratório: 01 Edema Agudo Pulmonar (EAP), 01 Insuficiência Respiratória Pulmonar Aguda (IRPA) e 01 laringo espasmo, nos outros 02 casos a reintubação ocorreu devido ao rebaixamento do nível de consciência. Destes pacientes que retornaram para a VM, 2 foram com menos de $2 \mathrm{~h}, 2$ com menos de $12 \mathrm{~h}$ e $1 \mathrm{com}$ menos de $24 \mathrm{~h}$.

Em média, os pacientes intubados (grupo 1) permaneceram 3,74 dias em VM antes da tentativa de retirada do respirador artificial.

No grupo 2, os pacientes foram submetidos ao procedimento de traqueostomia entre 7 a 14 dias de TOT, após a realização da traqueostomia a média de tempo de $\mathrm{VM}$ foi de 3,45 dias até 0 ato de retirada. $\mathrm{A}$ taxa dos que retornaram para VM foi de $40 \%(n=8)$. Sendo 03 em menos de $2 \mathrm{~h}, 04 \mathrm{com}$ menos de $12 \mathrm{~h}$ e $01 \mathrm{com}$ menos de $24 \mathrm{~h}$. A volta à VM neste grupo foi devido a esforço respiratório em todos casos $(n=8)$.

Quanto aos dados hemogasométricos colhidos no momento pré-extubação ou retirada da VM, obtivemos uma média dos valores da gasometria arterial nos grupos estudados. Subdividimos a média dos valores e desvio padrão nos casos de sucesso e nos casos de falha do processo de retirada da VM nos dois grupos (Tabela 1).

Tabela 1 - Conjunto de Médias, Desvio Padrão e T-Student dos dados hemogasométricos nos grupos de sucesso e falha no desmame.

\begin{tabular}{lccc}
\hline & Sucesso $\mathbf{n = 4 2}$ & Falha $\mathbf{n}=\mathbf{1 3}$ & Valor de $\mathbf{p}$ \\
\hline PH & $7,46 \pm 0,06$ & $7,49 \pm 0,04$ & 0,04 \\
PaO2 & $137,35 \pm 67,2$ & $144,15 \pm 66,2$ & 0,75 \\
PaCO2 & $41,02 \pm 6,90$ & $42,07 \pm 8,26$ & 0,70 \\
HCO3 & $29,61 \pm 6,71$ & $32,13 \pm 5,35$ & 0,17 \\
BE & $5,16 \pm 6,05$ & $6,84 \pm 3,90$ & 0,24 \\
Lactato & $1,15 \pm 0,63$ & $1,23 \pm 0,36$ & 0,59 \\
FIO2 & $45,71 \pm 6,67$ & $50,0 \pm 0$ & 0,0001 \\
P/F & $292,3 \pm 125,7$ & $288,3 \pm 132,4$ & 0,92 \\
\hline
\end{tabular}

Fonte: Dados da pesquisa, 2019.

Podemos observar que os valores médios de BE foram acima do valor de referência $(-2 \mathrm{a}+2 \mathrm{mmol} / \mathrm{L})$ nos dois grupos estudados, acompanhando os valores do $\mathrm{HCO}_{3}$. Os valores médios de lactato mantiveram entre 0,96 e $1,36 \mathrm{mmol} / \mathrm{L}$ colaborando com valores normais para retirada da VM nas amostras estudadas (Tabela 2). 
Tabela 2 - Valores máximos e mínimos dos dados hemogasométricos obtidos através da gasometria arterial nos pacientes com sucesso e falha no desmame:

\begin{tabular}{|c|c|c|c|c|c|c|c|}
\hline & & Max. & Mín. & & & Max. & Mín. \\
\hline & $\mathrm{PH}$ & 7.57 & 7.31 & & $\mathrm{PH}$ & 7,6 & 7,42 \\
\hline & $\mathrm{PO} 2$ & 304 & 57 & & PO2 & 299 & 73 \\
\hline \multirow[t]{3}{*}{ Grupo 1} & $\mathrm{PCO} 2$ & 87 & 30 & Grupo 2 & $\mathrm{PCO} 2$ & 55 & 32 \\
\hline & $\mathrm{HCO} 3$ & 48,6 & 16.1 & & $\mathrm{HCO} 3$ & 48.1 & 21,4 \\
\hline & $\mathrm{P} / \mathrm{F}$ & 608 & 146 & & $\mathrm{P} / \mathrm{F}$ & 598 & 114 \\
\hline \multicolumn{8}{|c|}{ Valores máx. e mín. - falha na retirada da VM } \\
\hline & & Max. & Mín. & & & Max. & Mín. \\
\hline \multirow{5}{*}{ Grupo 1} & $\mathrm{PH}$ & 7,55 & 7,45 & \multirow{5}{*}{ Grupo 2} & $\mathrm{PH}$ & 7,5 & 7,42 \\
\hline & $\mathrm{PO} 2$ & 235 & 82 & & PO2 & 254 & 85 \\
\hline & PCO2 & 55 & 32 & & PCO2 & 58 & 42 \\
\hline & $\mathrm{HCO} 3$ & 38,2 & 28 & & $\mathrm{HCO} 3$ & 43 & 25,9 \\
\hline & $\mathrm{P} / \mathrm{F}$ & 470 & 164 & & $\mathrm{P} / \mathrm{F}$ & 508 & 142 \\
\hline
\end{tabular}

Fonte: Dados da pesquisa, 2019.

Todos os pacientes com falha no processo de desmame no grupo 1 (TOT) apresentaram valor alcaloide no PH sanguíneo (acima de 7,45 ). No grupo 2, tanto no sucesso quanto na falha da retirada da VM não houve nenhum caso em que o PH sanguíneo ficou abaixo do valor de referência $(7,35)$ (Tabela 3).

Tabela 3 - Descrição dos dados hemogasométricos acima e abaixo dos valores de referência nas amostras de sucesso e falha da retirada da VM. Grupo 1- Pacientes Intubados; Grupo 2 - pacientes traqueostomizados.

\begin{tabular}{|c|c|c|c|c|c|c|}
\hline \multicolumn{7}{|c|}{ VALORES ACIMA DA NORMALIDADE NOS CASOS DE SUCESSO } \\
\hline \multirow{3}{*}{ Grupo 1} & & $\mathrm{PH}>7,45$ & PCO2 > 45 & $\mathrm{HCO} 3>26$ & $\mathrm{PO} 2>100$ & $P / F>300$ \\
\hline & $\mathbf{N}^{\circ}$ pacientes & 14 & 6 & 19 & 18 & 10 \\
\hline & $\%$ & $40 \%$ & $17 \%$ & $54 \%$ & $51 \%$ & $29 \%$ \\
\hline \multirow{2}{*}{ Grupo 2} & $\mathbf{N}^{\circ}$ pacientes & 8 & 3 & 10 & 8 & 7 \\
\hline & $\%$ & $66 \%$ & $25 \%$ & $83,3 \%$ & $67 \%$ & $58 \%$ \\
\hline \multicolumn{7}{|c|}{ VALORES ABAIXO DA NORMALIDADE NOS CASOS DE SUCESSO } \\
\hline \multirow{3}{*}{ Grupo 1} & & $\mathrm{PH}<7,35$ & PCO2 $<35$ & $\mathrm{HCO} 3<22$ & $\mathrm{PO} 2<80$ & $\mathrm{P} / \mathrm{F}<200$ \\
\hline & $\mathbf{N}^{\circ}$ pacientes & 3 & 5 & 1 & 4 & 8 \\
\hline & $\%$ & $9 \%$ & $14 \%$ & $3 \%$ & $11 \%$ & $23 \%$ \\
\hline \multirow[t]{2}{*}{ Grupo 2} & $\mathbf{N}^{\circ}$ pacientes & 0 & 3 & 1 & 3 & 2 \\
\hline & $\%$ & $0 \%$ & $25 \%$ & $8,33 \%$ & $25 \%$ & $17 \%$ \\
\hline \multicolumn{7}{|c|}{ VALORES ACIMA DA NORMALIDADE NOS CASOS DE FALHA } \\
\hline \multirow{3}{*}{ Grupo 1} & & $\mathrm{PH}>7,45$ & $\mathrm{PCO} 2>45$ & $\mathrm{HCO} 3>26$ & PO2> 100 & $P / F>300$ \\
\hline & $\mathbf{N}^{\circ}$ pacientes & 5 & 3 & 5 & 4 & 2 \\
\hline & $\%$ & $100 \%$ & $60 \%$ & $100 \%$ & $80 \%$ & $40 \%$ \\
\hline \multirow{2}{*}{ Grupo 2} & $\mathbf{N}^{\circ}$ pacientes & 6 & 1 & 7 & 5 & 2 \\
\hline & $\%$ & $75 \%$ & $12,50 \%$ & $87,5 \%$ & $62 \%$ & $25 \%$ \\
\hline \multicolumn{7}{|c|}{ VALORES ABAIXO DA NORMALIDADE NOS CASOS DE FALHA } \\
\hline \multirow{3}{*}{ Grupo 1} & & $\mathrm{PH}<7,35$ & $\mathrm{PCO} 2<35$ & $\mathrm{HCO} 3<22$ & $\mathrm{PO} 2<80$ & $\mathrm{P} / \mathrm{F}<200$ \\
\hline & $\mathbf{N}^{\circ}$ pacientes & 0 & 1 & 0 & 0 & 1 \\
\hline & $\%$ & $0 \%$ & $20 \%$ & $0 \%$ & $0 \%$ & $20 \%$ \\
\hline \multirow{2}{*}{ Grupo 2} & $\mathbf{N}^{\circ}$ pacientes & 0 & 0 & 0 & 1 & 3 \\
\hline & $\%$ & $0 \%$ & $0 \%$ & $0 \%$ & $12 \%$ & $37 \%$ \\
\hline
\end{tabular}

Fonte: Dados da pesquisa, 2019. 
Dos 6 pacientes com aumento do PCO2 no grupo 1, todos estavam com valor de HCO3 também aumentado. Dos 3 pacientes no grupo 2, também todos apresentavam valor acima da normalidade de HCO3.

\section{DISCUSSÃO}

A retirada da Ventilação Mecânica Invasiva (VMI) em terapia intensiva é um procedimento rotineiro devido uma grande parte dos pacientes necessitarem de suporte ventilatório durante o período de internação, a avaliação dos exames e clínica do paciente deve ser realizada de forma cautelosa e segura. Um estudo com uma amostra de 311 pacientes com quadro de traumatismo crânio encefálico no Hospital Geral do Estado da Bahia concluiu que em pacientes com Traumatismo Crânio Encefálico (TCE) a falha na extubação pode prolongar a permanência hospitalar, aumentar a frequência de traqueostomia, complicações pulmonares, piorar os resultados funcionais e aumentar a mortalidade (REIS HFC, et al., 2013).

Para o início do procedimento de desmame do paciente em VMI devemos avaliar todos os parâmetros possíveis em busca de executar o desmame da ventilação mecânica de formas mais segura e obter um menor índice de retorno a ventilação artificial (TEIXEIRA C, et al., 2012). Alguns estudos mostram que os valores gasométricos mínimos para se iniciar o desmame são: $\mathrm{PaO}_{2}>60 \mathrm{mmHg}$; $\mathrm{FiO} 2 \leq 40 \%$; SatO2 > 90\%; pH > 7,30 < 7,60; PaO2/FiO2 2200 e Peep < 5 (COLOMBO T, et al., 2007).

Em média as amostras de gasometria arterial deste estudo seguiram os parâmetros normais para início de desmame e retirada da VMI como $\mathrm{PH}, \mathrm{PAO} 2, \mathrm{PCO} 2, \mathrm{SpO} 2, \mathrm{PaO} 2 / \mathrm{FiO} 2$ e PEEP, exceto a $\mathrm{FIO} 2$ que a média foi acima do ideal entre $42 \%$ e $50 \%$ (Tabela 1), influenciando no valor de P/F da gasometria arterial, porém não foi obtido diferença no resultado do sucesso ou falha da retirada da VMI quanto a relação $P / F(p=$ 0,92).

Os melhores preditores para o sucesso da extubação são os valores do ânion gap sérico pré-extubação e a relação da pressão arterial parcial de oxigênio (Pao2) / fração inspirada de oxigênio (Fio2). Além disso, os valores mais baixos de $\mathrm{PaO} 2 / \mathrm{FiO} 2$ foram significativamente associados à falha de extubação $(P=0,032)$ (SAUGEL B, et al., 2018).

Os valores de PAO2 em média mantiveram acima da média (120 mmhg) em ambos os grupos (Tabela 1) não influenciando no sucesso ou falha da retirada da VM $(p=0,75)$, porém observamos um excesso do uso de oxigênio no desmame da VM mantendo valores de PAO2 muito altos chegando até $304 \mathrm{mmhg}$ (Tabela 2), como a maioria dos TRE foram em peça $T$, poderia ter sido realizado com níveis mais baixos de oxigênio adiantando assim o seu desmame minimizando efeitos colaterais e obtendo uma relação PAO2/FIO2 mais confiável. $O$ excesso de oxigênio pode causar toxicidade para as células do organismo humano e outras repercussões como a dependência e a narcose carbônica tendo assim que ser utilizado o mínimo necessário para manter uma SPO2 acima de 93\% e PaO2 acima de 80mmhg (BARBAS CSV, et al., 2013).

No estudo de Teixeira et al., (2012) foi feita uma pesquisa com 731 pacientes, buscou observar a eficácia de um protocolo de desmame na taxa de falha de extubação em pacientes com desmame difícil, obteve um índice de sucesso de extubação de $86,7 \%$ em um grupo com protocolo de desmame e $69,6 \%$ no grupo controle. Foi possível observar no estudo que os valores de $\mathrm{PaO} 2$ e $\mathrm{PaCO} 2$ não apresentaram diferenças relevantes em suas médias quando comparadas nos respectivos grupos, os dados obtidos foram estes (Tabela 4).

Tabela 4 - Médias de PaO2 e PaCO2 da amostra analisada:

\begin{tabular}{ccccccc} 
& \multicolumn{3}{c}{ GRUPO PROTOCOLO } & \multicolumn{3}{c}{ GRUPO CONTROLE } \\
\cline { 2 - 7 } & TODOS & SE & FE & TODOS & SE & FE \\
& $n=533$ & $n=462$ & $n=71$ & $n=198$ & $n=138$ & $n=60$ \\
$\mathrm{PaO}_{2}$ & $80+-23$ & $79+-20$ & $82+-26$ & $83+-19$ & $82+-20$ & $85+-16$ \\
$\mathrm{PaCO}_{2}$ & $32+-5$ & $31+-4$ & $33+-6$ & $32+-6$ & $31+-4$ & $33+-5$ \\
\hline
\end{tabular}

SE= Sucesso extubação; FE = Falha Extubação.

Fonte: Dados da pesquisa, 2019. 
Ao avaliarmos o $\mathrm{HCO} 3$ e o BE podemos observar que nos pacientes com sucesso de desmame no grupo 1 , os resultados permanecem levemente alcaloides em $54,2 \%$ dos casos. No grupo 2 cerca de $83,3 \%$ dos casos de sucesso mantiveram valores de $\mathrm{HCO} 3$ e $\mathrm{BE}$ elevados e $100 \%$ nos com falha no desmame mantiveram valores alcaloides moderados chegando a $48,1 \mathrm{mmol} / \mathrm{L}$ o $\mathrm{HCO} 3$ e $25,9 \mathrm{mmol} / \mathrm{L}$ o BE (Tabela 2).

Na Noruega, com uma amostra de 138.523 gasometrias arteriais de pacientes internados em CTI's afirmou que o distúrbio ácido-básico mais comum em pacientes de CTI é a alcalose metabólica. $O$ estudo relata também o aumento rápido nos valores de BE após a admissão em cerca de $85 \%$ das amostras analisadas e ressalta que embora a acidose seja mais comum na admissão no $\mathrm{CTI}$, o bicabornato aumenta com o tempo (MAEHLE K, et al., 2018).

Em um outro estudo que aborda a extubação de pacientes críticos, observou que durante todo o TRE do seu estudo o valor do bicabornato no grupo no qual a extubação falhou foi significativamente $(p<0,05)$ maior do que no grupo de sucesso na extubação. Colaborando com os resultados desta pesquisa em que os valores de bicabornato obtidos mantiveram valores alcaloides em $100 \%$ dos pacientes com falha na extubação, apesar do resultado estatístico não significativo $(p=0,11)$ (LIU L, et al., 2012; MAEHLE K, et al., 2018).

No grupo 2 por se tratar de amostras de pacientes traqueostomizados que necessitaram de maior tempo de ventilação mecânica acredita-se que a elevação do bicabornato foi influenciada pelo tempo de ventilação mecânica (Tabela 3).

$\mathrm{Na}$ insuficiência respiratória crônica hipercápnica e na acidose respiratória, o equilíbrio químico é interrompido e existem mecanismos fisiopatológicos que elevam os níveis de bicarbonato. A acidose respiratória associada ao aumento dos níveis de dióxido de carbono é compensada pelo aumento da excreção renal de ácido $\left(\mathrm{H}^{+}\right)$e retenção de bicarbonato. Esses mecanismos compensando a acidose respiratória e causando um aumento nos níveis de bicarbonato podem contribuir para uma redução dos valores do aníon gap sérico com pacientes em risco de falha na extubação, demonstrando que a hipercapenia pré-extubação está associada a alto risco de falha na extubação (SAUGEL B, et al., 2018).

Portanto, é importante ressaltar que se observarmos um aumento da PCO2 isso irá auxiliar a avaliar o uso da ventilação não invasiva após a extubação orotraqueal, principalmente em pacientes retentores crônicos de PCO2 como portadores de Doença Pulmonar Obstrutiva Crônica (DPOC) (LIU L, et al., 2012; MAEHLE K, et al., 2018).

Jeganatham N, et al. (2015) em sua pesquisa de revisão de literatura com o intuito de otimizar o valor e o desempenho do TRE de pacientes de alto risco para reduzir a taxa de falha na extubação, ressalta em sua conclusão que a realização de uma análise de gasometria arterial no final do TRE pode ajudar a identificar aqueles que poderiam se beneficiar da aplicação profilática da VNI imediatamente após a extubação (SAUGEL B, et al., 2018; MORTARI DM, et al., 2013).

Ao analisarmos a PCO2 em média os resultados permaneceram em valores normais e não obtivemos diferença significativa entre os 2 grupos $(p=0,70)$, assim como falha ou sucesso de retirada da VM (Tabela 1), no entanto observamos um aumento do bicabornato nos pacientes que estavam com PCO2 acima do valor de referência (Tabela 3 ).

Em um estudo realizado com 60 pacientes no Rio de Janeiro que teve como objetivo avaliar se existe um grupo de parâmetros que podem predizer os pacientes que irão ter o desmame da VM com sucesso assim como em nosso estudo, não encontrou valores significativos nos dados hemogasométricos em grupos de sucesso e insucesso do desmame (FREITAS EEC e DAVID CMN, 2006). O mesmo estudo ressalta ainda que a diferença em dias de internação no grupo com sucesso $(16,16 \pm 11,24)$ foi significativamente menor que no grupo com falha no desmame $(35,69 \pm 20,19 ; p=0,0001)$.

Na comparação de 4 métodos de desmame diferentes apesar de não apresentar valor estatisticamente significativo o $\mathrm{PaO} 2$ foi menor no grupo com menor índice de sucesso (ventilação por SIMV) quando comparados aos demais três grupos. Após o início do desmame, o maior valor de $\mathrm{PaO} 2, \mathrm{PaCO} 2, \mathrm{PH}$ foi no grupo NPPV (Non-invasive positive pressure ventilation) respectivamente [(98,92 $\pm 15,14),(47,2 \pm 5,55),(7,41$ 
$\pm 0,03)]$ e o menor valor foi no grupo SIMV, respectivamente [(89,52 $\pm 9,77),(49,92 \pm 4,79),(7,39 \pm 0,03)]$ (MABROUK AA, et al., 2015). Ainda neste estudo, outro dado sem diferença estatística relevante, mas com visível diferença nas médias quando divididos os grupos em falha e sucesso de desmame são os valores de $\mathrm{PaO} 2$ e $\mathrm{PaCO} 2$.

Tabela 5 - Média e Desvio padrão dos valores de PaO2 e PaCO2 nos grupos de sucesso e falha no desmame da VMI:

\begin{tabular}{lccc}
\hline & Sucesso & Falha & P value \\
\cline { 2 - 4 } $\mathrm{PaO} 2 \pm \mathrm{DP}$ & $137,35 \pm 67,29$ & $144,15 \pm 66,23$ & 0,75 \\
$\mathrm{PaCO} 2 \pm \mathrm{DP}$ & $41,02 \pm 9,87$ & $42,07 \pm 8,26$ & 0,70 \\
\hline
\end{tabular}

Fonte: Dados da pesquisa, 2019.

\section{CONCLUSÃO}

Esta pesquisa documental constatou que os valores de PAO2 e PCO2 da gasometria arterial no processo de desmame não interferiram no sucesso da retirada da ventilação mecânica, porém os dados hemogasométricos contribuíram de forma positiva no processo de desmame, o valor médio de $\mathrm{FiO} 2$ no grupo com sucesso foi significativamente menor que o valor médio de FiO2 no grupo com falha, portanto um valor alto de $\mathrm{FiO} 2$ pode influenciar nas taxas de insucesso do desmame. Dos que falharam todos mantiveram uma alcalose metabólica moderada demonstrando interferir negativamente no processo de desmame.

\section{REFERÊNCIAS}

1 SILVA GJP, et al. Estudo da prevalência de complicações pulmonares no pós-operatório de cirurgias cardíacas. Revista Brasileira de Fisioterapia. 2010. v. 14, p. 346.

2 VIESSER L. Fatores pré-analíticos que afetam as determinações de pH e gases sanguíneos. UNIVERSIDADE FEDERAL DO PARANÁ, CURITIBA 2012

3 CALDEIRA FM, WESTPHAL GA. Manual prático de medicina intensiva. São Paulo: Editora Segmento, 2006.

4 LIU L, et al. Neuroventilatory efficiency and extubation readiness in critically ill patients. Critical Care. 2012. 16: R143.

5 TEIXEIRA C, et al. Impacto de um protocolo de desmame de ventilação mecânica na taxa de falha de extubação em pacientes de difícil desmame. J. Bras. Pneumol. Porto Alegre. 2012. 38 (3): 364-371

6 COLOMBO T, et al. Implementação, avaliação e comparação dos protocolos de desmame com tubo-t e pressão suporte associada à pressão expiratória final positiva em pacientes submetidos à ventilação mecânica por mais de 48 horas em unidade de terapia intensiva. Revista Brasileira de Terapia Intensiva. v. 19, n. 1, p. 31-37. janeiro/março 2007.

7 SAUGEL B. et al. Prediction of extubation failure in medical intensive care unit patients. Journal of Critical Care. 2012. 27: 571-577. Disponível em: https://doi.org/10.1016/j.jcrc.2012.01.010. Acesso em 28 de dezembro de 2018.

8 BARBAS CSV, et al. Diretrizes de Ventilação Mecânica. AMIB e SBPT, I Fórum de Diretrizes em ventilação mecânica 2013

9 MAEHLE K, et al. Metabolic alkalosis is the most common acid-base disorder in ICU patients. Critical Care. Department of Anaesthesiology and Intesive Care of Nordland Hospital. Norway. 2014. 18:420.

10 MORTARI DM, et al. Prevalência de pacientes com indicação para uso de ventilação mecânica não-invasiva em uma unidade de emergência. Rev. Fac. Ciênc. Méd. Sorocaba. 2010. v.12, n.1, p. 13 - 16. UNILUS Ensino e Pesquisa. 2013

11 LAIZO A, et al. Complicações que aumentam o tempo de permanência na unidade de terapia intensiva na cirurgia cardíaca. Revista Brasileira de Cirurgia Cardiovascular. 2010. v. 25 (2), p. 166-171.

12 MABROUK AA, et al. Evaluation of some predictors for successful weaning from mechanical ventilation. Egyptian Journal of Chest Diseases an Tuberculosis. 2015. v. 64, p. 703-707.

13 CASSEL LW, VIEIRA SRR. Avaliação da intervenção fisioterapêutica em uma população geral de pacientes críticos submetidos a um protocolo de extubação orotraqueal. Dissertação de Mestrado apresentada no programa de pós-graduação em medicina. Universidade Federal do Rio Grande do Sul. 2013.

14 ROLIM JFC, et al. Variáveis hemodinâmicas, hemogasométricos e respiratórias em pacientes cardiopatas submetidos ao teste de respiração espontânea. Fisioter. Mov. Curitiba, v. 24, n. 4, p. 673-682, out./dez. 2011.

15 PINTO JMA, et al. Gasometria arterial: aplicações e implicações para enfermagem. Revista Amazônia Science \& Health. abril/junho 2017.v. 5, n. 2, p. 33 a 39

16 PAREDES ER, et al. Protocolo de prevenção de falha de extubação com estratégia para evitar as complicações da reintubação precoce. Revista UNILUS Ensino Superior. Vol. 10, $\mathrm{n}^{\circ} 19$ (2013).

17 JEGANATHAM N, KAPLAN CA, BALK RA. Ventilator liberation for high-risk-for-failure patients: Improving value of the spontaneous breathing trial. Rush University Medical Center and Rush Medical College, Chicago, Illinois - EUA. Respiratory Care. v. 60, n. 2. February 2015

18 REIS HFC, et al. Insuficiência da extubação influencia desfechos clínicos e funcionais em pacientes com traumatismo crânio encefálico. Jornal Brasileiro de Pneumologia. São Paulo. v. 39, n. 3. maio/junho 2013.

19 FREITAS EEC, DAVID CMN. Avaliação do sucesso do desmame da ventilação mecânica. Revista Brasileira de Terapia Intensiva. v. 18, n. 4, p. 351-359. outubro/dezembro 2006.

20 PIOTTO RF, et al. Efeitos da aplicação de protocolo de desmame de ventilação mecânica em unidade coronária: estudo randomizado. Revista Brasileira de Cirurgia Cardiovascular. São José do Rio Preto, SP. v. 26, n. 2. abril/junho 2011. 